McGill Law Journal

Revue de droit de McGill

\title{
L’héritage de l’affaire Roncarelli c. Duplessis 1959-2009
}

\section{Geneviève Cartier}

Volume 55, numéro 3, september 2010

The Legacy of Roncarelli v. Duplessis, 1959-2009

L'héritage de l'affaire Roncarelli c. Duplessis, 1959-2009

URI : https://id.erudit.org/iderudit/1000617ar

DOI : https://doi.org/10.7202/1000617ar

Aller au sommaire du numéro

Éditeur(s)

McGill Law Journal / Revue de droit de McGill

ISSN

0024-9041 (imprimé)

1920-6356 (numérique)

Découvrir la revue

Citer ce document

Cartier, G. (2010). L'héritage de l'affaire Roncarelli c. Duplessis 1959-2009.

McGill Law Journal / Revue de droit de McGill, 55(3), 375-400.

https://doi.org/10.7202/1000617ar d'utilisation que vous pouvez consulter en ligne. 


\section{L'HÉRITAGE DE L'AFFAIRE RONCARELLI C. DUPLESSIS 1959-2009}

\section{Geneviève Cartier*}

\section{Introduction : un cinquantenaire à souligner}

Le 27 janvier 1959, la Cour suprême du Canada rendait jugement dans l'affaire Roncarelli c. Duplessis ${ }^{1}$. Cette décision, et plus précisément l'opinion du juge Ivan Rand, a profondément marqué le droit public canadien. En droit constitutionnel, elle est principalement associée à l'émergence de la notion de primauté du droit, conçue comme un principe constitutionnel exerçant une contrainte effective sur l'action gouvernementale indépendamment de l'existence d'une disposition législative ou constitutionnelle formelle explicite, et à l'idée du common law bill of rights. En droit administratif, elle est systématiquement invoquée au soutien du principe selon lequel il n'existe rien de tel qu'un pouvoir discrétionnaire sans entraves et pour affirmer que le détenteur d'un pouvoir public ne peut en abdiquer l'exercice. À un autre niveau, elle est assimilée à la victoire d'un certain constitutionnalisme de common law à l'anglaise, typique du style du juge Rand.

Ces énoncés reflètent le sens et la portée qui sont aujourd'hui généralement attribués à l'affaire Roncarelli. Cependant, c'est sur la base de l'hypothèse suivant laquelle tout n'a pas été dit sur cette décision, et plus encore qu'elle pouvait inspirer de nouvelles avenues, que quatorze universitaires se sont réunis à la fin de l'été 2009 dans le cadre d'un symposium intitulé « L'héritage de l'affaire Roncarelli c. Duplessis-19592009-The Legacy of Roncarelli v. Duplessis $»^{2}$. Les participants ont

* Professeure titulaire, Faculté de droit de l'Université de Sherbrooke. Les textes qui composent ce numéro spécial de la Revue de droit de McGill se réfèrent à la présente introduction pour la présentation des faits de l'affaire et pour le résumé des jugements. Comme la plupart de ces textes sont en anglais, une version anglaise de cette introduction est disponible sur le site Web de la Revue (<http://lawjournal.mcgill.ca>).

(c) Geneviève Cartier 2010

Citation: (2010) 55 McGill L.J. $375 \sim$ Référence : (2010) 55 R.D. McGill 375

[1959] R.C.S. 121, 16 D.L.R. (2e) 689 [Roncarelli avec renvois aux R.C.S.].

2 La décision a en effet suscité très peu d'articles de doctrine depuis cinquante ans. Voir notamment E.C.S. Wade, Commentaire de Roncarelli c. Duplessis (C.S.), (1951) 29 R. du B. can. 665 ; Benjamin J. Greenberg, Commentaire de Duplessis c. Roncarelli, (1956) 3 R.D. McGill 82; Edward McWhinney, Commentaire de Roncarelli c. Duplessis 
profité du cinquantenaire de l'arrêt pour tenter de déboulonner quelques mythes, déterrer certains artéfacts, redécouvrir le familier ou ce qui semble appartenir au domaine de l'évidence. Les textes qui composent le présent numéro constituent le fruit de ce symposium.

La mission qui nous est confiée pour la présente introduction est double. D'une part, dans le but d'alléger la tâche des auteurs et pour éviter les redites, nous retraçons les faits de l'affaire et présentons les grandes lignes des nombreuses opinions judiciaires qu'elle a générées (I). D'autre part, nous présentons brièvement les travaux des participants, en esquissant quelques-uns des thèmes qui ont été discutés lors de notre rencontre (II).

\section{Les faits, les recours, les jugements : une saga à volets multiples}

À l'automne 1946, Frank Roncarelli exploite à Montréal un restaurant de très bonne réputation. Comme son père avant lui, Roncarelli détient un permis d'alcool délivré par la Commission des liqueurs du Québec (la Commission) et pendant près de trente-cinq ans, le permis de cette entreprise familiale a été renouvelé annuellement, sans interruption.

Citoyen apparemment sans histoire, Roncarelli est membre des Témoins de Jéhovah. Or, depuis quelques années, ce mouvement religieux indispose les autorités étatiques et ecclésiastiques québécoises. En effet, ses adeptes font montre d'un prosélytisme offensif et ils attaquent âprement la religion catholique, à laquelle la majorité de la population québécoise est alors profondément attachée, ce qui ira jusqu'à provoquer des émeutes dans certaines villes de la province ${ }^{3}$.

(C.S.C.), (1959) 37 R. du B. can. 503 ; Claude-Armand Sheppard, "Roncarelli v. Duplessis: Art. 1053 C.C. Revolutionized» (1960) 6 R.D. McGill 75, reproduit dans (2010) 55 R.D. McGill v; Luc Huppé, "Une immunité de poursuite civile pour les ministres» (1998) 32 R.J.T. 333 ; David J. Mullan, «Unwritten Constitutional Principles: The Legacy of Justice Rand ", Ivan C. Rand Memorial Lecture, University of New Brunswick, 2002 [non publiée]; David Dyzenhaus, "The Deep Structure of Roncarelli v. Duplessis » (2004) 53 R.D. U.N.-B. 111.

3 La réaction viscérale des autorités politiques et religieuses à la "propagande» des Témoins de Jéhovah s'explique certes par les méthodes employées par ses membres (porte-à-porte, distribution de documents dans les rues, etc.), mais également par la doctrine jéhoviste elle-même. Celle-ci prône en effet qu'une religion véritable ne doit pas entraver le rapport direct que les fidèles peuvent entretenir avec Dieu et dénonce donc le caractère résolument hiérarchique de la religion catholique. Le coup porte davantage encore compte tenu de la proximité alors entretenue entre l'Église et l'État et explique en grande partie les accusations de sédition portées contre des membres de la secte, notamment dans l'affaire Boucher c. R. ((1950), [1951] R.C.S. 265, [1951] 2 D.L.R. 369). Pour un exposé de la situation de l'époque, voir Michel Sarra-Bournet, L'affaire Roncarelli: Duplessis contre les Témoins de Jéhovah, Québec, Institut québécois de recherche sur la culture, 1986 aux pp. 41-72 [Sarra-Bournet, Roncarelli]; Thomas R. Berger, Liberté fragile : droits de la personne et dissidence au Canada, trad. par Marie- 
$\mathrm{Au}$ plus fort de la crise, en 1945 et 1946, la Ville de Montréal entreprend d'appliquer avec zèle un règlement qui exige un permis pour colporter et distribuer des publications afin de limiter l'impact des activités de la secte ${ }^{4}$. Les Témoins de Jéhovah sont d'avis qu'un tel règlement contrevient à leur liberté de culte et refusent de s'y conformer. Arrêtés par dizaines, ils sont remis en liberté en attendant leur procès grâce à Roncarelli, qui se porte caution sous la forme d'un engagement écrit ${ }^{5}$. En deux ans, plus de quatre cents membres bénéficieront de son geste. La plupart des membres ainsi libérés retournent à leur pèlerinage, mais ils sont à nouveau arrêtés puis libérés sous caution, créant un cycle qui a pour effet de congestionner les tribunaux et de monopoliser les forces de police. Constatant l'engorgement des tribunaux, les autorités décident d'exiger des cautions plus élevées, et en argent comptant. C'est ainsi qu'à compter du 17 novembre 1946, Roncarelli cesse de se porter caution.

Quelques jours plus tard, les Témoins de Jéhovah entreprennent au Québec une campagne intensive de distribution d'un véritable brûlot, $L a$ haine ardente du Québec pour Dieu, pour Christ, et pour la liberté, est un sujet de honte pour tout le Canada, qui dénonce leur persécution par les catholiques et qui en impute la responsabilité à la domination des prêtres ${ }^{6}$. Cette campagne se démarque des activités courantes de la secte par son extrême virulence et elle aura un effet incendiaire dans la province, à tel point que le 21 novembre 1946, lors d'une conférence de presse, le Premier ministre de l'époque, Maurice Duplessis, sert un avertissement solennel aux Témoins de Jéhovah. Ayant pris connaissance de La haine ardente, il affirme y avoir trouvé "certaines parties intolérables et vraiment séditieuses » et qu'en conséquence, il a "donné instruction à l'assistant-procureur général de prendre toutes les mesures

Cécile Brasseur en collaboration avec Daniel Séguin, Ville de LaSalle (Qc), Hurtubise HMH, 1985 aux pp. 171-91.

4 Dans les années 1930, la Ville de Québec avait déjà adopté un règlement similaire visant à limiter l'action des Témoins de Jéhovah en établissant des conditions contraignantes pour la distribution de leurs publications. Ce règlement fut invalidé par la Cour suprême du Canada. Voir Saumur c. Québec (Ville de), [1953] 2 R.C.S. 299, [1953] 4 D.L.R. 641.

5 Dans son témoignage, Roncarelli explique que ses cautionnements prennent la forme d'un engagement écrit et signé et non d'un versement en argent comptant. En vertu d'une entente avec les autorités judiciaires, Roncarelli en signe quelques-uns à l'avance, pour éviter d'avoir à se déplacer à la cour ou pour prévoir le cas où il serait absent de la ville au moment où une caution serait demandée. L'entente semble permettre aux autorités judiciaires de sortir de l'embarras, car toutes les personnes arrêtées ne peuvent être emprisonnées.

6 La haine ardente du Québec, pour Dieu, pour Christ, et pour la liberté, est un sujet de honte pour tout le Canada, Toronto, Watch Tower Bible and Tract Society, 1946 [La haine ardente]. Un certain Aimé Boucher sera un peu plus tard accusé de libelle séditieux en vertu du Code criminel (L.R.C. 1985, c. C-46) pour avoir distribué des copies de ce pamphlet. Voir Boucher, supra note 3. 
nécessaires pour découvrir les coupables et de ne pas hésiter à recourir aux moyens qui s'imposeront pour mettre fin à ces méthodes insupportables $» 7$.

C'est dans le cadre de cette chasse aux coupables que le gérant de la Commission, Édouard Archambault, apprend que Roncarelli détient un permis d'alcool ${ }^{8}$. Archambault communique alors avec Duplessis pour l'informer de son intention d'annuler le permis de Roncarelli, en vertu du pouvoir que lui délègue la Loi des liqueurs alcooliques d'annuler tout permis à sa discrétion ${ }^{9}$. Archambault affirme avoir obtenu de Duplessis "son consentement, son approbation, sa permission, et son ordre de procéder $»^{10}$. De son côté, Duplessis témoignera qu'il était de son " devoir, en conscience, de dire au Juge [Archambault] que [...] le Gouvernement de Québec ne pouvait pas accorder un privilège à un individu comme Roncarelli qui tenait l'attitude qu'il tenait»; qu'il a "approuvé la suggestion du g[é]rant général»; qu’il a dit à Archambault "Vous avez raison, ôtez le permis, ôtez le privilège "; et qu'en approuvant ainsi une suggestion comme supérieur, " c'est un ordre que l'on donne ${ }^{11}$.

Le permis est donc formellement annulé par Archambault, qui envoie sans délai des inspecteurs saisir l'alcool au restaurant de Roncarelli, sans aviser ce dernier de la mesure ni l'informer de ce qui lui est reproché. Le choc est cinglant pour le restaurateur. Le jour même, Duplessis s'adresse à la presse :

Le 21 novembre dernier, dans une déclaration faite aux courriéristes parlementaires, j'ai réitéré la ferme intention du gouvernement de Québec d'adopter les mesures les plus rigoureuses et les plus efficaces contre ceux qui sous le nom de Témoins de Jéhovah font une propagande répréhensible et distribuent des circulaires qui, dans mon opinion, sont non seulement injurieuses pour la province de Québec et sa population, mais revêtent, à notre avis, un caractère nettement séditieux. [...]

Un certain M. Frank Roncarelli s'est porté caution pour les Témoins de Jéhovah dans plusieurs centaines de cas. La sympathie que cet homme témoigne aux Témoins de Jéhovah — et cela d'une manière aussi évidente, multipliée et audacieuse — constitue une provocation

7 L'Action Catholique (21 novembre 1946) 3; Le Devoir (22 novembre 1946) 9, tels que cité dans Sarra-Bournet, Roncarelli, supra note 3 à la p. 40.

8 Linformation provient du procureur adjoint de la Couronne, Oscar Gagnon, à qui on a laissé entendre que Roncarelli était lié à la distribution du dépliant, notamment parce qu'une saisie de centaines de copies avait été effectuée dans un immeuble lui appartenant. C'est lorsqu'il apprit que Roncarelli détenait un permis d'alcool que Gagnon en était venu à communiquer avec Archambault.

$9 \quad$ Loi des liqueurs alcooliques, S.R.Q. 1941, c. 255, art. 35.

10 Roncarelli c. Duplessis (1951), [1952] 1 D.L.R. 680 à la p. 690 [Roncarelli (C.S.)].

11 Ibid. aux pp. 691-92. 
à l'ordre public, à l'administration de la justice dans la province et est absolument contraire aux fins de la justice. [...]

Aujourd'hui, ce monsieur Roncarelli s'identifie avec la propagande malsaine et odieuse des Témoins de Jéhovah. En conséquence, à titre de procureur général et de premier ministre, j'ai donné ordre d'annuler la licence accordée par la Commission des liqueurs, au restaurant exploité par cet homme à 1429, rue Crescent, à Montréal.

Les communistes, les nazis, ainsi que ceux qui se constituent les propagandistes de la campagne séditieuse des Témoins de Jéhovah, seront traités comme ils le méritent. Sous le gouvernement de l'Union nationale, il n'y a pas et il ne saurait y avoir de compromis avec ces gens-là ${ }^{12}$.

Roncarelli ne restera pas sans réagir. Il demande à A.L. Stein de le représenter et le presse d'approcher un certain Frank R. Scott, éminent professeur de droit de l'Université McGill et défenseur des libertés civiles $^{13}$.

Dans les mois qui suivent, Roncarelli doit surmonter plusieurs obstacles pour faire valoir ses droits. L'article 12 de la Loi des liqueurs alcooliques conditionne en effet le droit de poursuivre personnellement le gérant de la Commission "pour actes [...] accomplis ou omis dans l'exercice de ses devoirs " à l'autorisation du juge en chef de la province. Le juge Létourneau refuse cette autorisation, dans une décision du 5 février $1947^{14}$. Roncarelli sollicite alors le consentement du procureur général afin de poursuivre la Commission elle-même, comme l'exige la même disposition. Duplessis, qui occupe cette fonction à l'époque, ne lui répondra jamais directement: il annoncera publiquement son refus en conférence de presse, le 7 février $1947^{15}$. Il réaffirmera du même souffle sa

12 L'Action Catholique (4 décembre 1946) 9 ; Le Canada (5 décembre 1946) 14 ; Le Devoir (4 décembre 1946) 3, tels que cité dans Sarra-Bournet, Roncarelli, supra note 3 aux pp. 44-45.

13 Pour une biographie de Frank R. Scott, voir Sandra Djwa, F.R. Scott, une vie, trad. par Florence Bernard, Montréal, Boréal, 2001. Voir aussi Roderick A. Macdonald, « Francis Reginald Scott, 1899-1985» (1985) 30 R.D. McGill 635.

14 Roncarelli c. Archambault, [1947] B.R. 105. Dans sa décision, le juge indique que la disposition invoquée " est pour le cas où, abusant de ses fonctions, le gérant de la Commission des liqueurs aurait de mauvaise foi, par motifs pervers ou autrement, encouru personnellement la responsabilité civile prévue aux art. 1053 ou 1054 C.C.» (ibid. à la p. 107). Or, « la demande du requérant ne fait rien voir à ce sujet». En effet, "rien n'apparaît qui pût impliquer [la] responsabilité personnelle du juge Edouard Archambault" puisque les allégations ne se rapportent "qu'à des actes qu'aurait accomplis ledit gérant dans l'exercice de ses fonctions, mais nullement à des actes susceptibles d'engager ou d'en déduire sa responsabilité personnelle» (ibid.). Michel Sarra-Bournet rapporte qu'une nouvelle demande sera rejetée le 17 avril suivant. Voir Sarra-Bournet, Roncarelli, supra note 3 à la p. 49.

15 «Le procureur général adjoint, Édouard Asselin, statue, le 6 février 1947, que l'action de la Commission était conforme aux pouvoirs que la loi lui confère et, étant d'ordre administratif, ne tombe pas sous la juridiction de la Cour supérieure » (Sarra-Bournet, Roncarelli, supra note 3 à la p. 49). 
conviction que son geste était pleinement justifié, avant d'ajouter que " [1] permis a été cancellé et annulé, non pas temporairement, mais bien pour toujours ${ }^{16}$. Les procureurs de Roncarelli décident finalement de poursuivre Duplessis personnellement: A.V. Dicey n'enseigne-t-il pas qu'en vertu du principe de la primauté du droit, tous sont égaux devant la $\operatorname{loi}^{17}$ ? Mais le temps presse : non seulement le recours se prescrit-il par six mois ${ }^{18}$, mais encore faut-il donner à Duplessis un avis de trente jours avant de procéder, en vertu de l'article 88 du Code de procédure civile qui l'exige dans le cas de poursuites en dommages intentées contre un officier public « à raison d'un acte par lui fait dans l'exercice de ses fonctions". Le 3 juin 1947, sans avoir pu se conformer à l'article 88 C.p.c., Roncarelli intente une action en dommages-intérêts pour annulation illégale de permis contre Duplessis personnellement. Treize ans plus tard, la Cour suprême du Canada lui donnera raison.

Lorsque la Cour rend jugement en 1959, quinze juges répartis sur trois niveaux d'instance se sont prononcés dans cette affaire et pas moins de douze d'entre eux ont rédigé leur propre opinion ${ }^{19}$. Pourtant, l'affaire Roncarelli sera rapidement presque exclusivement associée à l'opinion du juge Rand. Les raisons qui peuvent expliquer la prééminence de cette dernière sont multiples et les contributions qui suivent en suggèrent de fort intéressantes. Nous verrons cependant que l'exhumation des autres opinions exprimées dans cette affaire permet non seulement de procéder à une analyse plus profonde et plus nuancée de sa décision, mais jette aussi un nouvel éclairage sur le contexte sociopolitique de l'affaire, les défis procéduraux qui l'ont jalonnée, la complexité des rapports qu'elle soulève entre le droit public et le droit privé et l'émergence d'une conception particulière du constitutionnalisme.

16 L'Action catholique (8 février 1947) 3, tel que cité dans Sarra-Bournet, Roncarelli, supra note 3 à la p. 49.

17 A. V. Dicey, Introduction to the Study of the Law of the Constitution, 10e éd., Londres, Macmillan \& Co., 1959 à la p. 193 : " [N]o man is above the law, [...] [E]very man, whatever be his rank or condition, is subject to the ordinary law of the realm and amenable to the jurisdiction of the ordinary tribunals ".

18 Voir Loi des privilèges des juges de paix et des magistrats, S.R.Q. 1941, c. 18, art. 5 : "Aucune [...] action [en dommages-intérêts] ou poursuite ne peut être intentée contre un juge de paix, un officier ou toute autre personne [remplissant des devoirs publics], pour un acte qu'ils ont fait dans l'exécution de leurs devoirs publics, à moins qu'elle ne soit commencée dans les six mois qui suivent la commission de l'infraction". Pour bénéficier de la protection de la loi, les officiers doivent être de bonne foi «bien qu'en faisant un acte, ils aient excédé leurs pouvoirs ou leur juridiction, et aient agi clairement contre la loi » (ibid., art. 7).

19 De tous les juges qui se sont prononcés sur l'affaire, seuls les juges de la Cour suprême du Canada Judson et Locke, qui souscrivent respectivement à l'opinion de leurs collègues Rand et Martland, ne fournissent pas leurs propres motifs. Le juge en chef Kerwin, qui se joint lui aussi à Martland, se contente de quelques brefs commentaires. 


\section{A. Une victoire en Cour supérieure du Québec}

Roncarelli obtient gain de cause en Cour supérieure du Québec. Le juge Mackinnon conclut que Duplessis a bel et bien ordonné à Archambault de révoquer le permis d'alcool de Roncarelli et que cet ordre a été le facteur déterminant de la décision prise par Archambault ${ }^{20}$. Selon le juge, on ne peut blâmer Duplessis pour avoir considéré le pamphlet $L a$ haine ardente comme étant de nature séditieuse et pour avoir tenté d'en limiter la distribution, mais Duplessis ne pouvait viser Roncarelli pour atteindre indirectement le mouvement religieux dans son ensemble ${ }^{21}$. Par ailleurs, au terme d'une étude attentive des textes législatifs pertinents ${ }^{22}$, aucune source formelle n'autorisait Duplessis à s'immiscer dans le processus entourant l'octroi ou l'annulation des permis d'alcool. Quant à la Commission, elle a agi arbitrairement en annulant le permis pour une raison étrangère à la réglementation relative aux permis d'alcool (l'appartenance de Roncarelli aux Témoins de Jéhovah et son rôle de caution) et au mépris des règles de la raison et de la justice (" disregarded the rules of reason and justice " $)^{23}$. Le juge ajoute qu'étant donné les principes de droit anglais selon lesquels "acts done in their official character but in excess of their lawful authority ${ }^{24}$ induisent la responsabilité de ceux qui les posent, Duplessis doit répondre de ses actes comme un simple citoyen. Le juge Mackinnon affirme enfin que Duplessis ne peut plaider l'absence de l'avis prescrit par l'article 88 C.p.c. puisqu'il s'impose lorsque l'officier agit dans l'exercice de ses fonctions et non, comme en l'espèce, à l'occasion de celles-ci ${ }^{25}$.

\section{B. Un recul en Cour du Banc de la Reine}

La Cour du Banc de la Reine renverse la décision de la Cour supérieure du Québec à quatre juges contre un, essentiellement sur une interprétation différente de la preuve présentée. En effet, pour les juges Bissonnette, Pratte, Casey et Martineau, la preuve établit que la Commission avait déjà pris la décision d'annuler le permis lorsqu'elle a consulté Duplessis ${ }^{26}$ et qu'il n'y a donc aucun lien de causalité entre l'acte de ce dernier et la décision de la Commission, ce qui est fatal à l'issue du

Roncarelli (C.S.), supra note 10 à la p. 692.

Ibid. à la p. 682.

Ibid. aux pp. 697-99.

Ibid. à la p. 695. Le juge paraphrase Lord Halsbury dans Sharp v. Wakefield, [1891] A.C. 173 à la p. 179, tel que cité dans Roncarelli (C.S.), supra note 10 à la p. 694.

Albert Venn Dicey, Law of the Constitution, 1939 aux pp. 193-94, tel que cité dans Roncarelli (C.S.), supra note 10 à la p. 696.

Ibid. à la p. 700.

Duplessis c. Roncarelli, [1956] B.R. 447 aux pp. 455 (juge Bissonnette), 466 (juge Pratte), 485 (juge Martineau) [Roncarelli (C.A.)]. 
recours. Si le juge Pratte se limite à cette conclusion, ses collègues Bissonnette, Martineau et Casey poursuivent la discussion avec une analyse du bien-fondé de la décision de la Commission.

Pour imputer une faute à Duplessis, affirment les juges Bissonnette et Martineau, il faudrait d'abord démontrer que la Commission a elle-même exercé sa discrétion de manière fautive ou illégale et ensuite, estime Martineau, que Duplessis était de mauvaise foi ${ }^{27}$. Ils affirment qu'en l'espèce, la bonne foi de ce dernier ne fait aucun doute, pas plus que la légalité de la décision de la Commission. Pour le juge Bissonnette, la Commission est un organisme "essentiellement commercial» et les détenteurs de permis, "[s]es serviteurs »28. Il lui était donc " loisible" de prononcer l'annulation du permis sans qu'un tribunal ne puisse en vérifier le fondement ${ }^{29}$. En l'espèce, ajoute le juge, le gérant de la Commission "s'il estimait que les agissements du demandeur étaient subversifs et constituaient un mal que ses pouvoirs permettaient de supprimer, avait la faculté, plus que cela, le devoir d'intervenir efficacement $»^{30}$. Pour le juge Martineau, le commerce des liqueurs est "licite mais non libre", le permis " un privilège " et la Commission dispose d'une discrétion " quasi illimité[e] " pour le révoquer, discrétion qui est seulement contrainte par la bonne foi ${ }^{31}$. En l'espèce, Duplessis s'était conduit de manière raisonnable à la lumière des "normes d'un homme typique, normal, du lieu et du temps où l'acte a été fait $»^{32}$. Dans le contexte de décembre 1946, en effet, l'attitude de Roncarelli constituait " une provocation qui aurait enlevé tout élément de faute à l'ordre de révoquer le permis du demandeur ${ }^{33}$. Le juge Casey affirme quant à lui que la Commission doit exercer sa discrétion " in accordance with what [it] believes to be the public interest and welfare $»^{34}$. Il s'ensuit que, si la Commission avait des motifs raisonnables de croire que les Témoins de Jéhovah menaient une campagne de nature séditieuse, Roncarelli se rendait indigne du privilège en tant que membre actif de la secte ${ }^{35}$. La Commission avait par conséquent le devoir d'annuler le permis, si bien qu'en ordonnant à la

\footnotetext{
27 Ibid. aux pp. 456-57 (juge Bissonnette), 482 (juge Martineau).

28 Ibid. à la p. 457.

29 Ibid.

$30 \quad$ Ibid. à la p. 458.

31 Ibid. à la p. 482.

$32 \quad$ Ibid. à la p. 491.

33 Ibid. à la p. 490.

34 Ibid. à la p. 470.

35 Ibid. à la p. 471.
} 
Commission de le faire, Duplessis ne faisait que lui enjoindre d'accomplir son devoir de sorte qu'aucune faute ne peut lui être imputée ${ }^{36}$.

Dissident, le juge Rinfret ne voit aucune erreur qui justifie de remettre en cause l'interprétation des faits par la Cour supérieure du Québec: la révocation du permis résulte bel et bien de l'ordre de Duplessis ${ }^{37}$. Or, aucune disposition législative ne confère à ce dernier un droit de surveillance sur la Commission ${ }^{38}$. De son côté, la Commission a commis une illégalité en agissant sous les ordres d'un tiers puisque le détenteur d'un pouvoir discrétionnaire doit l'exercer lui-même ${ }^{39}$. Le juge reconnait que ces considérations permettent de disposer du litige, mais il poursuit.

Premièrement, bien que la discrétion d'accorder, de refuser ou de renouveler un permis soit absolue, puisqu'« aucun droit tangible $»^{40}$ n'est mis en cause, la discrétion d'annuler un permis pendant sa durée de validité n'est pas sans limites. Dans un tel cas, il existe certainement un "droit quelconque [...] si infime et aléatoire soit-il» auquel il ne peut être porté atteinte que pour des raisons liées à la loi qui accorde le pouvoir discrétionnaire, et en conformité avec les principes qui doivent en guider l'exercice ${ }^{41}$. L'exercice du pouvoir doit s'accorder avec les objectifs pour lesquels le pouvoir a été délégué, il doit reposer sur des faits vérifiés, être exercé après avoir permis à la personne de se défendre des accusations portées contre elles et tenir compte de considérations pertinentes ${ }^{42}$. Deuxièmement, pour pouvoir répondre à un mouvement qu'il pouvait légitimement croire séditieux, Duplessis devait se limiter aux "moyens mis à sa disposition par la loi et les statuts »43. Or, il a emprunté "à un autre des moyens de répression que la loi lui refuse ${ }^{44}$. Au surplus, une vérification des faits aurait convaincu Duplessis que rien ne reliait Roncarelli à la sédition présumée. Duplessis ne pouvait pas non plus justifier son geste par le fait qu'il s'inscrivait dans le cadre plus large de la campagne de suppression des activités des Témoins de Jéhovah. Pour le juge Rinfret, "frapper une personne contre qui rien de tangible n'est établi pour en atteindre une autre $»^{45}$ mine encore davantage la légitimité de l'acte posé par Duplessis.

Ibid. à la p. 475 .

Ibid. aux pp. 502-503.

Ibid. à la p. 506.

Ibid. à la p. 507.

Ibid. à la p. 508.

Ibid.

Ibid. à la p. 507.

Ibid. à la p. 511.

Ibid. à la p. 512.

Ibid. à la p. 515 . 


\section{La Cour suprême du Canada et le juge Rand}

Le juge Martland ${ }^{46}$ est d'avis que l'annulation du permis résulte d'un ordre de Duplessis, qui n'agissait sur la base d'aucune justification juridique. En effet, rien dans la loi ne l'autorisait à agir et son mandat de procureur général ne lui conférait aucune liberté « to use any methods he chooses; that, on suspicion of participation in what he thinks would be an offence, he may sentence a citizen to economic ruin without trial ${ }^{47}$. Le juge Martland considère que cette question doit être déterminée en droit et non sur la base de la bonne foi ou de l'appréciation personnelle que fait l'officier public de ses fonctions ${ }^{48}$. Or, rien ne permettait à Duplessis de croire qu'il était autorisé à priver le demandeur de son permis. Il n'agissait donc pas dans l'exercice de ses fonctions lorsqu'il l'a fait et ne peut exiger l'avis requis par l'article 88 C.p.c.

Le juge ajoute par ailleurs que la compétence statutaire de la Commission d'annuler un permis à sa discrétion suppose l'existence d'un rapport entre les raisons invoquées pour agir et l'intention et le but de la loi ${ }^{49}$. Elle doit être exercée sans la soumission à un tiers ${ }^{50}$. Or, l'association de Roncarelli aux Témoins de Jéhovah et le paiement par lui de plusieurs cautions n'avaient rien d'illégal et étaient étrangers à l'intention et au but de la loi. De plus, la preuve indique que la Commission a agi sous la dictée de Duplessis. Le juge conclut que Duplessis a intentionnellement causé le dommage, sans justification légale, ce qui entraîne sa responsabilité 51 .

Le juge Abbott partage cette lecture de la preuve, des motifs d'annulation et de l'absence de lien entre ces motifs et les buts et objectifs de la Loi des liqueurs alcooliques ${ }^{52}$. De toute façon, ajoute le juge, aucune disposition législative n'autorisait Duplessis à intervenir dans les décisions de la Commission, un organisme indépendant. Le fait qu'il ait été convaincu que son geste s'inscrivait dans ce qu'il concevait comme les meilleurs intérêts du peuple, n'a aucune pertinence sur la question de sa responsabilité en dommages pour actes causés à la suite d'un excès de compétence: "[T]he respondent was acting without any legal authority whatsoever. Moreover, [... he] was bound to know that he was acting with-

$$
\begin{aligned}
& \text { Voir Djwa, supra note } 13 \text { à la p. } 436 . \\
& 48 \text { Roncarelli, supra note } 1 \text { à la p. } 158 . \\
& 49 \text { Ibid. à la p. } 156 . \\
& 50 \quad \text { Ibid. à la p. } 157 . \\
& 51 \quad \text { Ibid. à la p. } 159 . \\
& 52 \quad \text { Ibid. à la p. } 184 .
\end{aligned}
$$
Duplessis aurait effectivement clairement indiqué en cours d'audience devant la Cour que l'objectif visé par Duplessis était de miner la situation économique de Roncarelli. 
out such authority "53. C'est également cet élément qui empêche Duplessis de bénéficier de l'exigence de l'article 88 C.p.c. ${ }^{54}$.

Quant au juge Fauteux, il fonde entièrement sa dissidence sur ce dernier article, tout en indiquant, cependant, que n'eût été cette disposition, il aurait décidé en faveur de Roncarelli. En effet, affirme-t-il, bien qu'on ne puisse douter de la bonne foi de Duplessis et du gérant de la Commission, ce dernier a abdiqué son pouvoir de décider et Duplessis, " comme Premier Ministre et Procureur Général, [...] s'est arrogé un droit que lui nie virtuellement la Loi des Liqueurs Alcooliques; il a commis une illégalité ${ }^{55}$ qui donne droit à réparation.

Toutefois, l'article 88 C.p.c. règle la question: disposition de nature prohibitive dont l'irrespect emporte nullité en vertu du Code civil du Bas Canada, elle limite la juridiction du tribunal, puisque l'absence d'avis empêche tout verdict ou jugement d'être rendu ${ }^{56}$. La question de savoir si l'acte reproché à Duplessis a été fait "dans l'exercice de ses fonctions " doit trouver une réponse dans la perspective suivant laquelle l'article 88 C.p.c. accorde un traitement procédural particulier aux officiers publics et son application suppose précisément que l'officier a commis une illégalité, sans égard à la bonne ou mauvaise foi de ce dernier ${ }^{57}$. En l'espèce, Duplessis a commis une illégalité, sans que sa bonne foi ne soit mise en cause, et il a commis cette illégalité à cause de sa fonction ${ }^{58}$. Il doit donc bénéficier de la protection de l'article 88 C.p.c.

La dissidence du juge Taschereau repose sur des bases similaires. Comme c'est à titre de procureur général, et non à titre personnel, qu'Archambault a consulté Duplessis, et même si celui-ci a agi sur la base d'une conception erronée de son devoir public, son acte conservait néanmoins son caractère officiel et se situait «dans l'exercice de ses fonctions $»^{59}$.

Également dissident, le juge Cartwright affirme que Duplessis ne sera responsable des dommages que si l'annulation du permis était « an actionable wrong ${ }^{60}$, ce qui n'est pas le cas. La preuve établit que Duplessis croyait honnêtement remplir son devoir en ordonnant l'annulation du permis d'un individu qui utilisait les avantages d'un privilège pour miner les lois existantes. Dans la mesure où cette opinion "was honestly 
entertained", aucun tribunal ne peut vérifier si la preuve appuyant cette opinion est suffisante, ni si cette opinion constitue un motif raisonnable d'annulation ${ }^{61}$. En effet, le législateur a conféré à la Commission une discrétion absolue qu'il n'a encadrée d'aucune règle, déléguant par là une fonction de nature administrative. Or, le détenteur de ce type de fonction est « a law unto itself $»^{62}$. Le juge Cartwright conclut donc qu'il n'y a aucun recours pour les dommages subis puisque l'annulation du permis découlait d'un acte de la Commission autorisé par la loi. Étant donné que l'acte de la Commission ne constitue pas une faute, il s'ensuit que Duplessis ne peut être tenu responsable des dommages pour avoir ordonné ou approuvé cet acte. Il est donc inutile d'aborder l'argument lié à l'article 88 C.p.c. ${ }^{63}$.

Le juge Rand ${ }^{64}$ insiste sur le fait que c'est comme simple citoyen, membre d'un groupe religieux, détenteur d'un permis d'alcool et fournissant caution dans le cadre de procédures liées à des infractions municipales que Roncarelli s'est retrouvé impliqué dans cette affaire. En ordonnant à la Commission d'annuler le permis de Roncarelli, Duplessis souhaitait non seulement mettre un terme à la campagne de propagande des Témoins de Jéhovah, mais aussi punir Roncarelli pour le rôle qu'il y avait tenu et servir un avertissement aux autres détenteurs de privilèges ${ }^{65}$. La question qu'il formule résume sa lecture des événements : "[W]hen the de facto power of the Executive over its appointees at will to such a statutory public function is exercised deliberately and intentionally to destroy the vital business interests of a citizen, is there legal redress by him against the person so acting? " ${ }^{66}$.

Le juge Rand souligne qu'un nombre grandissant d'activités nécessitent désormais un permis, à l'égard duquel les détenteurs deviennent dépendants au fil du temps, puisque ce permis conditionne bien souvent leur capacité à gagner leur vie ${ }^{67}$. La Commission se présente ainsi comme un service public qui doit gérer les permis de manière entièrement impartiale et intègre, ce qui implique que la discrétion de la

61 Ibid.

62 Re Ashby, [1934] O.R. 421 à la p. 428, [1934] 3 D.L.R. 565 (C.A.), tel que cité dans Roncarelli, supra note 1 à la p. 167.

Rona àne le juge Rand a conclure que la Commission a porté atteinte au droit de détenteur un détenir un privilege puisque le fait d'avoir un permis cree chez son interférence non autorisée. Voir ibid. à la p. 143. 
Commission de refuser un permis ou de l'annuler « is to be based upon a weighing of considerations pertinent to the object of the administration $» 68$.

Dans un des passages emblématiques de sa décision, le juge Rand affirme :

In public regulation of this sort there is no such thing as absolute and untrammelled "discretion", that is that action can be taken on any ground or for any reason that can be suggested to the mind of the administrator; no legislative Act can, without express language, be taken to contemplate an unlimited arbitrary power exercisable for any purpose, however capricious or irrelevant, regardless of the nature or purpose of the statute. Fraud and corruption in the Commission may not be mentioned in such statutes but they are always implied as exceptions. "Discretion" necessarily implies good faith in discharging public duty; there is always a perspective within which a statute is intended to operate; and any clear departure from its lines or objects is just as objectionable as fraud or corruption. Could an applicant be refused a permit because he had been born in another province, or because of the colour of his hair? The ordinary language of the legislature cannot be so distorted.

To deny or revoke a permit because a citizen exercises an unchallengeable right totally irrelevant to the sale of liquor in a restaurant is equally beyond the scope of the discretion conferred. There was here not only revocation of the existing permit but a declaration of a future, definitive disqualification of the appellant to obtain one: it was to be "forever". This purports to divest his citizenship status of its incident of membership in the class of those of the public to whom such a privilege could be extended. Under the statutory language here, that is not competent to the Commission and a fortiori to the government or the respondent [...]. [W] hat could be more malicious than to punish this licensee for having done what he had an absolute right to do in a matter utterly irrelevant to the Liquor Act? Malice in the proper sense is simply acting for a reason and purpose knowingly foreign to the administration, to which was added here the element of intentional punishment by what was virtually vocation outlawry69.

Il ajoute plus loin :

"Good faith" [...] means carrying out the statute according to its intent and for its purpose; it means good faith in acting with a rational appreciation of that intent and purpose and not with an improper intent and for an alien purpose; it does not mean for the purposes of punishing a person for exercising an unchallengeable right; it does not mean arbitrarily and illegally attempting to divest a citizen of an incident of his civil status ${ }^{70}$.

Quelle que soit l'immunité de la Commission, affirme le juge, Duplessis n'en a aucune: il est intervenu sans autorisation dans les

Ibid. à la p. 140.

69 Ibid. aux pp. 140-41.

70 Ibid. à la p. 143. 
fonctions de la Commission et a commis une faute qui engage sa responsabilité ${ }^{71}$. Il ajoute qu'à une époque de croissance de la réglementation administrative de l'activité économique, "that an administration according to law is to be superseded by action dictated by and according to the arbitrary likes, dislikes and irrelevant purposes of public officers acting beyond their duty, would signalize the beginning of disintegration of the rule of law as a fundamental postulate of our constitutional structure $»^{72}$.

\section{Le bilan d'un héritage}

Les circonstances qui ont mené à l'affaire Roncarelli forment une toile de fond indissociable des enjeux qu'elle a soulevés. Le Québec des années quarante, profondément catholique, est dirigé par un premier ministre dont la conception du pouvoir repose essentiellement sur la légitimité de toute action visant à préserver la culture et la spécificité de la nation canadienne-française, au prix de moyens parfois audacieux, frôlant souvent le mépris des institutions publiques ${ }^{73}$. Ce sont aussi les premiers balbutiements de l'État providence, ainsi que l'apprentissage des rapports entre les pouvoirs politique et exécutif. En 1945 et 1946, c'est enfin une série de confrontations entre les pouvoirs publics (municipaux et provinciaux) et les Témoins de Jéhovah dont le prosélytisme attise la colère de la majorité religieuse.

Si les circonstances que nous venons de décrire permettent d'expliquer la naissance du conflit entre Roncarelli et Duplessis, le jugement de la Cour suprême du Canada, qui est celui qui a laissé sa marque comme précédent, est lui aussi le produit de son époque. La composition de la Cour, notamment la présence du juge Rand, et les questions nouvelles qui l'occupent au lendemain de la Seconde Guerre mondiale constituent le terreau dans lequel pourront croître des idées nouvelles ou tout simplement s'en exprimer d'anciennes mais de manière renouvelée.

La maturation de cette décision dans les décennies qui ont suivi n'a pas été sans connaître des périodes prolongées de léthargie. Par ailleurs, les fruits qu'elle a produits ne ressemblaient pas nécessairement à ceux que prédisait, au lendemain de la décision rendue par la Cour suprême du Canada, Claude-Armand Sheppard dans un article publié dans la Revue de droit de McGill74. Dans les faits, la décision peut apparaître comme

$71 \quad$ Ibid. aux pp. 141-42.

72 Ibid. à la p. 142.

73 La question de l'héritage de Duplessis et de son association à la période qualifiée de " Grande Noirceur » a fait l'objet de multiples études. Voir notamment Alain-G. Gagnon et Michel Sarra-Bournet, dir., Duplessis : entre la grande noirceur et la société libérale, Montréal, Éditions Québec Amérique, 1997.

Sheppard, supra note 2. 
précurseur (de concert avec les décisions de l'époque associées à l'émergence du common law bill of rights) d'un constitutionnalisme qui ne prendra racine que plusieurs années plus tard, et paradoxalement, peutêtre, après l'adoption de la Charte canadienne des droits et libertés ${ }^{75}$.

L’impulsion ayant mené à la tenue du symposium reposait à la fois sur des travaux antérieurs, sur notre expérience d'enseignement et, plus précisément, sur la difficulté de démêler l'écheveau des diverses opinions, prétentions et conclusions de Roncarelli. Il nous semblait que, loin d'être une simple curiosité historique ${ }^{76}$, Roncarelli pouvait contribuer à la résolution des défis juridiques contemporains ou, au moins, à une meilleure compréhension de ces défis. Nous verrons brièvement comment les contributions des participants ont confirmé la pertinence de l'aventure. Nous présentons succinctement leurs travaux, en les regroupant plus ou moins librement à l'intérieur de trois grands thèmes qui ont retenu l'attention lors de nos discussions en groupe : des défis méthodologique et épistémologique (A) ; un double contraste entre simplicité et complexité, et entre théorie et pratique (B) ; et la prééminence de l'opinion du juge Rand (C).

\section{A. Des défis méthodologique et épistémologique}

La question de la méthode appropriée à l'étude d'un précédent, tout comme celle de savoir s'il est possible d'identifier ce qu'il a réellement décidé, ne sont pas spécifiques à l'affaire Roncarelli. Les défis méthodologique et épistémologique liés à la connaissance de la jurisprudence figurent parmi les préoccupations centrales des théories de l'interprétation. Cependant, les cinq décennies qui nous séparent du jugement à l'étude ont eu pour effet de projeter ces questions à l'avantplan des discussions lors du symposium. Peut-on prétendre établir ce que Roncarelli a véritablement décidé en 1959 ? Si oui, comment procéder ? Si non, la décision peut-elle avoir des significations différentes selon les lecteurs? Plus globalement, quel rapport peut-on ou doit-on entretenir avec cette décision cinquante ans plus tard ? De manière prévisible, ni les textes, ni les discussions n'ont fourni de réponses définitives à ces questions, mais nous pouvons constater que certaines contributions mettent l'accent sur la reconstitution de la décision, alors que d'autres insistent sur sa relecture. Ces deux perspectives ne sont pas totalement étrangères puisque, tout en y accordant une importance variable, elles prennent toutes deux au sérieux l'idée que la décision n'est pas totalement construite par l'interprète et que les faits qui en constituent l'origine sont importants.

75 Partie I de la Loi constitutionnelle de 1982, constituant l'annexe B de la Loi de 1982 sur le Canada (R.-U.), 1982, c. 11.

76 Contra Yves-Marie Morissette, «Rétrospective et prospective sur le contentieux administratif» (2008) 39 R.D.U.S. 1 à la p. 5. 
1. La reconstitution : le rapport au temps et le rapport au contexte

Une meilleure connaissance du contexte sociohistorique de la décision nous permet de reconstituer la décision et de mieux comprendre ce qu'elle a pu exprimer au moment où elle a été rendue. Dans cette lignée, la contribution de Roderick A. Macdonald 77 avance qu'une telle analyse contextuelle permet une interprétation de Roncarelli plus nuancée que celle véhiculée par le discours dominant. D'une part, il trace un profil sociodémographique et idéologique des juges des trois instances qui ont rendu jugement dans cette affaire, notamment quant aux aspects liés à la langue, à la religion et aux rapports entre le Québec et le reste du Canada. Cette analyse suggère que le clivage entre les juges majoritaires et minoritaires ne peut s'expliquer de manière simple, à travers une dichotomie représentant les premiers comme les défenseurs de la primauté du droit et les seconds comme concédant l'existence d'une sphère politique arbitraire inatteignable par le droit. Les multiples influences et profils des juges dans cette affaire et l'analyse des décisions qu'ils ont rendues suggèrent que les fondements de leurs décisions sont plus complexes qu'il n'y paraît.

D'autre part, Macdonald soutient que l'argumentation mise de l'avant par Duplessis en 1946 s'appuyait sur des théories sociales, politiques et juridiques solidement articulées et qu'une étude attentive de ces théories indique qu'elles auraient tout aussi bien pu constituer l'assise de la conclusion de la Cour suprême du Canada. En d'autres termes, sur la base des assises théoriques invoquées par Duplessis, la Cour aurait pu se demander s'il avait violé un devoir fiduciaire d'administrer la loi conformément à ses buts et pour le bénéfice de l'ensemble de la population visée par la réglementation, et conclure par l'affirmative. Toutefois, une telle condamnation n'aurait pas été due à la faiblesse des théories invoquées, mais au contexte factuel qui ne donnait pas raison à Duplessis. Pour Macdonald, le peu d'intérêt porté par les commentateurs aux arguments de ce dernier a contribué à radicaliser la lecture de la décision, en associant Duplessis à une position moralement et juridiquement intenable. De plus, en faisant abstraction du temps et du lieu dans lesquels elle a été rendue, les commentateurs lui ont attribué des conclusions que le contexte ne permettait pas de soutenir.

Dans la même veine, Eric M. Adams ${ }^{78}$ insiste lui aussi sur le fait que les précédents prennent leur sens à la lumière du contexte dans lequel ils naissent et dans lequel ils croissent. Il reconstitue ce contexte pour démontrer que le sens et la portée qui ont été attribués à Roncarelli résultent d'un processus de construction complexe, au terme duquel la

77 Voir Roderick A. Macdonald, « Was Duplessis Right?» (2010) 55 R.D. McGill 401.

78 Voir Eric M. Adams, «Building a Law of Human Rights: Roncarelli v. Duplessis in Canadian Constitutional Culture» (2010) 55 R.D. McGill 437. 
décision est devenue, pour un temps du moins, un jugement portant sur les droits fondamentaux. Non seulement la décision a-t-elle été rendue à l'époque de la naissance d'une culture constitutionnelle des droits, mais la division de la Cour dans l'affaire et la difficulté d'en identifier la ratio auraient favorisé une lecture de la décision propre à ne retenir que les éléments liés à la nature constitutionnelle des droits et à la citoyenneté, des thèmes alors intensément débattus au Canada. En éclipsant du paysage interprétatif la question de la responsabilité civile, qui constituait pourtant le point central de l'affaire, de même que le recours au principe de la primauté du droit pour justifier les limitations à l'autorité de la sphère exécutive de l'État, des processus de construction du sens et d'influences mutuelles entre juristes, universitaires, citoyens et autres acteurs sociaux ont propulsé la culture des droits et consacré la stature du juge Rand dans l'imaginaire canadien. Adams souligne à juste titre que cette construction n'est pas statique et qu'un processus similaire peut certainement expliquer pourquoi la décision n'a plus aujourd'hui le même attrait.

Derek McKee ${ }^{79}$ démontre combien la dichotomie entre les sphères publique et privée est fondamentale dans Roncarelli. À son avis, en énonçant le principe selon lequel tout exercice de pouvoir "public", incluant le pouvoir de nature discrétionnaire, fait l'objet de limites, le juge Rand s'appuyait sur une conception de la dichotomie public/privé caractéristique du libéralisme économique et politique du dix-neuvième siècle et d'une pensée juridique classique. McKee relève les multiples manifestations de la dualité public/privé dans l'affaire Roncarelli, comme par exemple, l'insistance de Duplessis à qualifier le permis de privilège, le rendant du même coup vulnérable à l'autorité publique. À l'inverse, le juge Rand refuse d'aborder la question sous l'angle droits/privilèges et insiste plutôt sur les intérêts d'affaires de Roncarelli de même que sur les conséquences économiques d'une révocation, faisant ici appel à une sphère privée que l'État ne peut légitimement pénétrer. Pour McKee, cette approche néglige le fait que la définition des contours d'une des sphères affecte nécessairement l'autre sphère et qu'en limitant la protection aux exercices de pouvoirs publics, Roncarelli laisse le pouvoir privé incontestable. Ainsi, le prestige de Roncarelli, associé à sa détermination à limiter l'arbitraire dans l'exercice du pouvoir public, doit aussi être vu comme la réaffirmation d'une sphère privée, que le pouvoir judiciaire peut difficilement contrôler.

2. La relecture : des théories contemporaines qui éclairent sa pertinence

Peut-on penser que la pleine signification d'un texte ancien émerge seulement progressivement, au fur et à mesure qu'il est interprété dans

79 Voir Derek McKee, «The Public/Private Distinction in Roncarelli v. Duplessis» (2010) 55 R.D. McGill 461. 
des contextes nouveaux et différents? Que la signification normative de la décision continue de se révéler en même temps que l'on réfléchit à ce qu'elle évoque? La façon dont les idées ont été exprimées dans le passé sont importantes. Macdonald, Adams et McKee ont, à ce chapitre, grandement contribué à nous faire mieux comprendre, grâce à une véritable remise en contexte, sur les plans social, historique et politique, ce qu'avait tenté d'exprimer la décision. Par ailleurs, les contributions au symposium ont aussi permis de voir l'intérêt de théories juridiques qui se sont developpées depuis les cinquante dernières années. Ainsi, certaines théories encore peu développées à l'époque de la décision de la Cour suprême du Canada peuvent aujourd'hui en faire émerger une compréhension nouvelle.

En ce sens, David Dyzenhaus ${ }^{80}$ affirme que des décisions comme Roncarelli acquièrent leur pérennité par leur capacité à éclairer d'une lumière nouvelle notre situation contemporaine. Il suggère que la lecture d'un extrait d'article écrit par Rand en $1960^{81}$ combinée à celle de son opinion dans Roncarelli révèlent les principaux éléments d'une théorie juridique républicaine. La théorie républicaine postule que la liberté individuelle se définit par l'absence de domination, par l'État ou par d'autres individus, par opposition à la liberté libérale, associée à l'idée de liberté en tant qu'absence d'interférence. Plus précisément, Dyzenhaus soutient que les écrits du juge Rand nous permettent de comprendre pourquoi la meilleure interprétation d'un constitutionnalisme de common law consiste à voir cette dernière comme une doctrine qui protège la liberté dans le sens républicain de non-domination. En utilisant le droit comme instrument de domination, c'est-à-dire comme un moyen de soumettre Roncarelli à son bon vouloir, Duplessis agissait sans égard à la question de savoir ce qui pouvait servir les intérêts de Roncarelli. L'autorité du droit est l'expression publique de notre droit à nous gouverner nous-mêmes et par conséquent de notre droit, à titre de sujets du droit, de ne pas être soumis à l'arbitraire des hommes, mais seulement aux lois qui servent notre intérêt dans la non-domination.

Evan Fox-Decent ${ }^{82}$ affirme que la vision du droit public qui soustend l'opinion du juge Rand affecte notre compréhension de la légalité de l'action administrative de même que les débats entourant le constitutionnalisme de common law. Le juge Rand soutient que les pouvoirs publics sont soumis à une obligation juridique d'exercer leur discrétion sans arbitraire, obligation qui découle d'un principe de légalité, auquel Rand confère le statut de principe constitutionnel non écrit. Le

80 Voir David Dyzenhaus, « Rand's Legal Republicanism» (2010) 55 R.D. McGill 491.

81 Ivan C. Rand, « Some Aspects of Canadian Constitutionalism» (1960) R. du B. can. 135.

82 Voir Evan Fox-Decent, «Democratizing Common Law Constitutionalism » (2010) 55 R.D. McGill 511. 
principal défi consiste à expliquer pourquoi les décideurs investis de pouvoirs discrétionnaires, qu'aucune loi statutaire ne limite expressément, sont soumis à une obligation juridique de n'exercer ces pouvoirs que pour des motifs limités. En effet, reconnaître que l'élimination de l'arbitraire est une valeur ou même un devoir en common law, n'en fait pas nécessairement une obligation juridique qui s'exerce indépendamment d'une disposition législative expresse à cet effet. Comment combler le fossé qui sépare valeur et obligation juridique? De l'avis de Fox-Decent, cela est possible dans le cadre d'une conception relationnelle et démocratique du droit public, qui est précisément ce qu'exprime le raisonnement du juge Rand dans Roncarelli. Cette conception est relationnelle dans la mesure où les acteurs publics et les individus soumis à leur pouvoir entretiennent des rapports de nature fiduciaire, qui excluent les rapports de domination et d'instrumentalisation. C'est la nature fiduciaire de ces rapports qui fait naître l'obligation juridique d'agir sans arbitraire. Cette conception du droit public est également démocratique en ce que son articulation prend sérieusement en compte les aspects concrets des rapports fiduciaires, tels qu'ils sont vécus par les acteurs. Pour Fox-Decent, une telle théorie fournit un fondement solide et une structure à la fois à la vision du droit public exprimée par le juge Rand dans l'affaire Roncarelli et à la place que ce dernier réserve à la discrétion dans le cadre du principe de la primauté du droit.

Matthew Lewans ${ }^{83}$ cherche de son côté à identifier les sources d'inspiration du juge Rand dans la formulation du type particulier de constitutionnalisme qui a fait la marque de sa décision. Il explique que c'est la notion de citoyenneté qui permet d'expliquer le caractère normatif de son modèle constitutionnel et que sa conception de la citoyenneté est largement inspirée du droit américain. Plus spécifiquement, Lewans montre que le juge Rand a tenté d'intégrer en droit canadien les principes d'égalité et de non-discrimination originellement visés par la clause privileges and immunities du quatorzième amendement de la Constitution des États-Unis, mais rapidement abandonnés par les juges majoritaires de la Cour suprême de ce pays. Lewans suggère que les fortes dissidences de la Cour américaine revivent dans la pensée du juge Rand. Tout en évoquant le caractère complexe de la citoyenneté et ses multiples éléments, Lewans relève que le principe d'égalité, dans ses volets positif et négatif, est particulièrement important pour Rand. Ce dernier combine les droits sociaux et économiques, se traduisant par exemple dans la nécessité de permettre l'accès aux " privilèges » de la citoyenneté, comme les permis d'alcool, sans discrimination (le volet positif) au droit à la noninterférence étatique dans les libertés individuelles, dont la liberté de religion (le volet négatif). Lewans admet que le bilan de la carrière du juge

83 Voir Matthew Lewans, Roncarelli's Green Card: The Role of Citizenship in Randian Constitutionalism » (2010) 55 R.D. McGill 537. 
Rand est moins reluisant en matière d'immigration, où sa conception de la citoyenneté paraît fort discutable, mais il souligne que le juge Rand doit être compris à la lumière du contexte et des idées de son temps. Étant donné que le contexte est important, son évolution a permis à des décisions comme celle de la Cour suprême du Canada dans l'affaire Baker c. Canada (Ministre de la Citoyenneté et de l'Immigration) ${ }^{84}$ de puiser à même l'opinion du juge Rand dans Roncarelli pour donner une impulsion nouvelle à sa conception caractéristique du constitutionnalisme.

Mark D. Walters ${ }^{85}$ suggère qu'il existe deux approches conceptuelles au principe de la primauté du droit : la primauté du droit comme raison et la primauté du droit comme ordre (respectivement legality as reason et legality as order). La première approche cherche à décrire les conditions particulières dans lesquelles la gouvernance s'effectue grâce au droit, c'est-à-dire les règles et les conditions que le droit doit remplir pour constituer un système de règles. La seconde s'intéresse plutôt à la façon dont la primauté du droit s'exprime à travers une forme d'interprétation justificative qui vise la cohérence, la cohésion et l'égalité, soit un processus dynamique de justification raisonnée. Walters suggère tout d'abord une lecture de la position du juge Rand comme combinant non seulement les deux aspects conceptuels de la primauté du droit, mais aussi leur interprétation doctrinale, d'une manière telle que le juge Rand enrichit notre compréhension du constitutionnalisme et permet de la valoriser concrètement, dans la pratique. Il soutient également que Dicey, compris à travers certains passages de textes inédits, dont sa correspondance personnelle, n'est peut-être pas si loin de la vision conceptuelle de la primauté du droit proposée par le juge Rand, alors que ces deux personnages sont traditionnellement présentés comme incarnant des positions antagoniques sur la question.

\section{B. Un double contraste : entre simplicité et complexité - entre théorie et pratique}

$\mathrm{Au}$ tout début de ce texte, nous avons relevé les quelques grands principes auxquels le jugement Roncarelli est traditionnellement associé. Pourtant, les participants au symposium ont rapidement souligné la très grande complexité de cette affaire, évoquant le contraste qu'elle établit avec l'apparente simplicité et la clarté des principes qui lui sont attribués (1). Dans une perspective similaire, certains ont dressé un bilan pour le moins mitigé de l'impact des leçons de l'affaire Roncarelli: le ton péremptoire sur lequel le juge Rand les a servies tranche en effet avec les hésitations du pouvoir judiciaire à les mettre en pratique. Ainsi, le

84 [1999] 2 R.C.S. 817, 174 D.L.R. (4e) 193.

85 Voir Mark D. Walters, «Legality as Reason: Dicey, Rand, and the Rule of Law " (2010) 55 R.D. McGill 563. 
contrôle judiciaire demeure absent de bien des lieux de pouvoirs discrétionnaires et la prévention de l'arbitraire tarde à s'inscrire dans les préoccupations des institutions judiciaires elles-mêmes (2).

\section{Le mirage de la simplicité}

La complexité du cas se manifeste à plus d'un égard : les procédures alambiquées auxquelles Roncarelli a dû recourir pour obtenir le droit de poursuivre ${ }^{86}$; les faits âprement contestés; la nature essentiellement privée du recours et le caractère public du défendeur ainsi que du cadre juridique dans lequel plusieurs des arguments ont été présentés; l'importance accordée par les juges à l'analyse de la décision de la Commission, alors que le recours visait Duplessis ; le nombre, l'ampleur et le style des opinions rédigées par les juges ayant entendu l'affaire, à tous les niveaux d'instance. En résumé, Roncarelli présente une allure kaléidoscopique, que Mullan, Aronson et Bernatchez ont bien mis en évidence.

Dans une analyse minutieuse et nuancée, David Mullan ${ }^{87}$ aborde ce qui est peut-être le plus intrigant dans l'affaire Roncarelli. En effet, le litige se présentait sous la forme d'une action en responsabilité civile et, au lendemain du jugement, Sheppard se disait d'avis que la position de la Cour sur la notion de faute constituait l'élément central de la décision en ce qu'elle révolutionnait l'état du droit sur la question ${ }^{88}$. Cet aspect de la décision a pourtant été rarement discuté par la suite ${ }^{89}$. À travers son analyse, qui évoque entre autres le jeu des influences entre la common law et le droit civil, Mullan tente de décrypter les diverses opinions de la Cour pour comprendre ce qu'elle a "véritablement » décidé sur la question du fondement de la condamnation de Duplessis. Du même coup, Mullan démontre la difficulté de concilier les opinions des juges des trois instances, mais avance qu'une majorité des juges de la Cour suprême du Canada considéraient que la simple illégalité suffit à engendrer une faute. Mullan montre également comment cette règle s'est subséquemment modifiée pour exiger la preuve de mauvaise foi du décideur. Son analyse illustre le défi que représente la compréhension d'ensemble de la décision et met en évidence que, sur le fond, les juges de l'époque étaient majoritairement favorables à une conception large de la responsabilité des décideurs publics, de laquelle le droit contemporain tend à prendre ses distances.

Voir à ce sujet le rappel des faits de l'affaire dans la partie I, ci-dessus. What Did It Stand in 1959 and For What Does It Stand in 2009?» (2010) 55 R.D. McGill 587.

Voir toutefois Huppé, supra note 2. 
Mark Aronson ${ }^{90}$ nous présente une perspective australienne de l'affaire Roncarelli, à travers une analyse de la portée du principe de la primauté du droit et des conditions de responsabilité des pouvoirs publics. D'une part, il souligne que les juges australiens auraient été fort réticents à déployer tel quel le principe de légalité pour condamner l'attitude de Duplessis. En effet, le droit australien ne reconnait aucune force normative directe à ce principe, qui est invoqué de manière essentiellement rhétorique, par des juges qui conçoivent majoritairement leur rôle à travers une approche légaliste. Par ailleurs, non seulement le droit statutaire de l'époque conférait-il des protections juridiques importantes au bénéfice des détenteurs de permis, mais plusieurs précédents établissaient des limites à l'exercice du pouvoir discrétionnaire, parmi lesquelles plusieurs auraient pu fonder une conclusion favorable à Roncarelli. D'autre part, Aronson souligne que, du point de vue australien, l'aspect le plus étonnant de l'affaire Roncarelli réside dans la condamnation effective du Premier ministre à des dommages-intérêts, sur la base de la primauté du droit et de règles relatives à la responsabilité qui revêtent un aspect distinct de celles qui s'appliquent au droit privé, alors que l'Australie rebute à procéder à une telle distinction. Au terme d'une étude attentive des tendances jurisprudentielles canadienne et australienne, Aronson constate que le droit canadien semble se diriger vers la reconnaissance d'une notion floue de "comportement public" moralement condamnable, alors que le droit australien semble vouloir se limiter à sanctionner l'abus de "pouvoir public", en des termes minimalistes qui visent à maintenir la vision " privée » de la responsabilité des pouvoirs publics.

Dans une perspective un peu différente, Stéphane Bernatchez ${ }^{91}$ avance que le recours au principe constitutionnel non écrit de la primauté du droit marque le début d'un mouvement qu'il qualifie de "procéduralisation réflexive" des dispositifs de gouvernance démocratique. D'une part, ce mouvement exprime le fait que les principes (comme celui de la primauté du droit) sont susceptibles de provoquer une remise en question des règles d'un système juridique, et des changements à ces dernières, le cas échéant. D'autre part, comme ces principes sont souvent associés à des dispositifs institutionnels favorisant la participation des acteurs (comme le droit d'être entendu), ces derniers participent à la construction du sens des normes auxquelles ils sont soumis. Par ailleurs, Bernatchez soutient que l'affaire Roncarelli peut être vue comme le point de départ de la «bifurcation" du droit administratif

90 Voir Mark Aronson, «Some Australian Reflections on Roncarelli v. Duplessis» (2010) 55 R.D. McGill 615.

91 Voir Stéphane Bernatchez, «Les rapports entre le droit administratif et les droits et libertés : la révision judiciaire ou le contrôle constitutionnel ? » (2010) 55 R.D. McGill 641. 
vers la logique des droits et libertés. À travers une analyse de la jurisprudence de la Cour suprême du Canada sur la question des concepts d'atteinte minimale et d'accommodement raisonnable, il met en lumière la complexité des rapports entre le droit constitutionnel et le droit administratif, et la difficulté corrélative d'identifier la voie à privilégier dans des circonstances où les deux domaines semblent se prêter au règlement d'un litige. La contribution de Bernatchez a suscité une remise en question plus large : comment distinguer le droit constitutionnel du droit administratif ? Et surtout peut-être, pourquoi les distinguer?

\section{Une mission inachevée}

Sossin et Liston proposent une mesure de l'écart qui sépare la théorie énoncée dans l'affaire Roncarelli et la pratique qui en a découlé. Tous deux mesurent le travail à accomplir.

Pour Lorne Sossin ${ }^{92}$, l'affirmation du juge Rand selon laquelle il n'existe rien de tel qu'un pouvoir discrétionnaire sans entraves au sein du pouvoir exécutif, représente l'héritage le plus important de l'affaire Roncarelli en même temps qu'une affirmation éloquente du principe de la primauté du droit. À son avis, toutefois, cet héritage est menacé par une interprétation judiciaire discutable de la doctrine de la justiciabilité, qui a pour effet d'isoler du contrôle judiciaire d'importants segments de pouvoirs discrétionnaires de l'exécutif. Sossin soutient que la jurisprudence en matière de justiciabilité doit être réévaluée à la lumière du principe de la primauté du droit, de manière à réaffirmer qu'aucune espèce particulière de discrétion n'est exemptée de contrôle judiciaire. Par ailleurs, la justiciabilité ne devrait pas être conçue sur le modèle du tout ou rien, mais bien plutôt sur celui du spectre, qui régit déjà le degré d'intervention à exercer en matière de contrôle judiciaire. De l'avis de Sossin, ce type de contrôle est un rempart nécessaire contre l'arbitraire, mais il ne suffit pas à assurer la concrétisation du principe énoncé dans Roncarelli : il faut encore que le respect de la primauté du droit résulte d'une impulsion interne à l'Administration. Cette dernière doit donc développer une culture interne de légalité à travers des démarches d'éducation, de supervision et de mentorat. La pleine réalisation des promesses de Roncarelli en dépend.

Mary Liston ${ }^{93}$ s'applique à définir la notion d'arbitraire qui, bien qu'occupant une place centrale dans l'affaire Roncarelli, n'y est pas explicitement articulée. Liston aborde trois dimensions de l'arbitraire (subjective, fonctionnelle et sociologique), qu'elle applique à l'analyse de la

92 Voir Lorne Sossin, «The Unfinished Project of Roncarelli v. Duplessis: Justiciability, Discretion, and the Limits of the Rule of Law » (2010) 55 R.D. McGill 661.

93 Voir Mary Liston, «Witnessing Arbitrariness: Roncarelli v. Duplessis Fifty Years On» (2010) 55 R.D. McGill 689. 
décision. Outre que Duplessis avait manifesté un arbitraire "subjectif» en faisant fi du principe de légalité, Liston souligne que, sur le plan fonctionnel, le droit ou ses failles institutionnelles faisaient problème: délégation de pouvoirs discrétionnaires excessivement étendus, absence d'indépendance du décideur, absence d'audition et de motivation de la décision, difficultés d'avoir accès aux tribunaux, etc. Liston se concentre par la suite sur un aspect étonnant de l'affaire: les diverses manifestations de l'arbitraire judiciaire. Elle souligne notamment sur ce plan la faiblesse de la motivation des opinions de certains juges, le fait que des questions n'aient pas été abordées et la confusion provoquée par les nombreux obiters. Liston conclut que la notion d'arbitraire, telle qu'elle émane de Roncarelli, continue d'exercer une force positive dans la consolidation $\mathrm{du}$ droit public canadien. Elle souligne cependant l'importance de relire la décision pour en faire ressortir les éléments qui n'ont pas été largement étudiés, de manière à éviter un affaiblissement de sa portée. Par ailleurs, elle affirme que la tâche visant à éliminer l'arbitraire est loin d'être complétée: en font foi les rapports qu'entretiennent les pouvoirs publics à l'égard de la protection des droits des Premières Nations. Liston affirme que l'héritage de Roncarelli est riche, mais, comme Sossin, elle s'empresse d'ajouter que la mission n'est pas terminée.

\section{La prééminence de l'opinion du juge Rand}

Nous avons suggéré plus tôt le fait que l'affaire Roncarelli est rapidement devenue "l'opinion du juge Rand». Comment expliquer cette prééminence? Plusieurs des textes qui composent le présent ouvrage relèvent la solidité de ses arguments ainsi que la force de sa rhétorique, mais la prédominance du jugement Rand n'est peut-être pas sans lien avec sa capacité pour l'abstraction : il arrive à distiller l'essence du litige et à le situer dans un ensemble plus élevé et plus large de principes, facilitant du même coup leur application ou leur discussion subséquentes.

Quel effet cette prédominance produit-elle? La réduction de l'analyse de l'affaire Roncarelli à l'opinion du juge Rand a eu deux effets principaux. Tout d'abord, elle a relégué à l'arrière-plan des considérations qui sont apparues à tort comme secondaires ou techniques, ce que démontre Leckey. Ensuite, elle a privé ce jugement d'une source de contrastes susceptibles de lui donner encore plus de poids et de valeur. C'est ce que Dyzenhaus a démontré en réponse aux arguments de Leckey.

Robert Leckey ${ }^{94}$ affirme la nécessité de réviser la lecture dominante de l'affaire Roncarelli, qui réduit cette dernière à une dualité simpliste

94 Voir Robert Leckey, «Complexifying Roncarelli’s Rule of Law» (2010) 55 R.D. McGill 721. 
entre les juges porte-étendards du principe de la primauté du droit, et les autres, formalistes et étroits d'esprit, ayant décidé de l'affaire sur la base de l'article 88 C.p.c. À travers une analyse minutieuse, Leckey décortique l'opinion du juge Fauteux et soutient que son approche est tout aussi compatible avec le principe de la primauté du droit que celle adoptée par le juge Rand. Leckey met en lumière la solidité des arguments présentés par le juge Fauteux et la perspective particulière dont il bénéficie à titre de juge de droit civil dans l'interprétation d'une telle disposition. Selon Leckey, les désaccords entourant l'interprétation de cet article constituent donc un débat interne à la primauté du droit, qui oblige les tenants de la position du juge Rand à expliquer comment le principe de légalité les avait amenés, dans les circonstances, à donner à une disposition législative explicite une interprétation qui s'éloignait de celle suggérée à la fois par son libellé et par la jurisprudence québécoise. Il en résulte une lecture de la décision beaucoup plus riche que celle que lui attribuent traditionnellement les universitaires.

Si le travail de Leckey replace dans une perspective plus juste l'opinion de Rand, il a également pour effet de permettre aux supporteurs de ce dernier de bonifier leur position en cherchant à répondre aux objections. Ainsi, Dyzenhaus est d'accord avec Leckey sur la structure de l'argument, mais considère que, sur le fond, le principe de la primauté du droit énonce qu'en l'absence d'une mention expresse en ce sens, une disposition législative ne peut être interprétée comme protégeant les décideurs publics des conséquences de leurs actes commis de mauvaise foi, de manière arbitraire ou par esprit de vengeance.

\section{Conclusion : une discussion à poursuivre}

Les thèmes abordés dans l'affaire Roncarelli sont d'une importance fondamentale pour toute communauté politique qui adhère à l'idéal d'un État de droit et qui s'interroge sur les rôles légitimes respectifs du législateur, du pouvoir exécutif et des instances judiciaires. Ils abordent les principes fondateurs de notre droit public : la question de la nature et de la portée des limites susceptibles d'encadrer l'exercice des pouvoirs publics de manière à préserver la liberté individuelle, et la mesure dans laquelle ils peuvent devoir compenser les dommages qu'ils causent.

Par ailleurs, ces questions sont non seulement récurrentes, elles sont particulièrement actuelles. À l'époque de Roncarelli, la montée de l'État providence et ses répercussions sur les individus exigeaient une relecture des principes juridiques développés pour un État gendarme. Aujourd'hui, les préoccupations sont doubles: non seulement l'État providence conserve-t-il sa place (malgré le discours qui plaide pour une réduction de la taille de l'État), mais les enjeux typiquement associés à l'État gendarme refont surface, dans la foulée des événements du 11 septembre 2001. Ainsi, les inquiétudes reliées à la sécurité nationale se traduisent 
fréquemment par un recours au pouvoir discrétionnaire par les plus hautes instances étatiques (comme les certificats de sécurité) et elles nous poussent à retourner aux sources de notre droit public et aux enseignements de Roncarelli.

$$
* * *
$$

« Il vient un temps où l'esprit aime mieux ce qui confirme son savoir que ce qui le contredit, où il aime mieux les réponses que les questions. Alors l'instinct conservatif domine, la croissance spirituelle s'arrête $»^{95}$. Loin de mener à un bilan définitif de l'héritage de l'affaire Roncarelli, nos travaux nous ont plutôt permis, cinquante ans plus tard, d'en mesurer la profondeur, la complexité et la pertinence. La discussion demeure donc ouverte, histoire de mettre en échec "l'instinct conservatif » au profit de l'enrichissement de l'esprit.

95 Bachelard, La formation de l'esprit scientifique, Paris, Librairie philosophique J. Vrin, 2004 à la p. 17. 